\title{
A Distributed Remote Real-time Pressure Measuring System Based on Wireless Communication Technology
}

\author{
http://dx.doi.org/10.3991/ijoe.v11i5.4654 \\ Wang W, Zhang Z J \\ North University of China, Taiyuan City, China
}

\begin{abstract}
With urgent requirements on the transient pressure test in the explosion field, the number of test nodes is increasing, which puts higher requirements on centralized management and control as well as process monitoring. A distributed measuring system, based on wireless communication technology, can manage and control all test nodes scattered in the testing area through a wireless network, realizing scatter operation, collaboration and centralized control, and thus is a key research point in today's distributed measuring field. Based on the basic principles of the distributed testing, this paper designs a wireless distributed measuring system used for pressure measurement of explosion fields. The central control can realize long-distance management, controlling and monitoring of many test nodes through a wireless local network, including the working parameter configuration of the each node in the testing preparation stage; the working state monitoring reset of each node in the waiting for explosion stage and so on; and the real-time transmission of the testing data.
\end{abstract}

Index Terms-burst-mode transmission, distributed testing, wireless local area network

\section{INTRODUCTION}

In the damage effectiveness evaluation of a weapon system, the transient pressure of the shock wave is an important method to directly test the blast effect, which is of great importance to the study of damage effect and personal protection [1]. Traditionally, people use storage testing technology to test shock waves, but with the urgent requirements of transient pressure in the explosion field and the updating explosion equivalent, the testing environment is becoming more complicated and is in demand of several testing equipment layouts in the explosion field. In this testing, the reading of the data after the explosion, the state monitoring the test equipment before the explosion, and the management of the system require the testing staff's cooperation, which can lower the efficiency of the testing. Recently, because of the need for distributed testing of explosion fields and the wide application and continual development of the distributed testing system, the researchers are combining a distributed testing system with a design transient pressure testing system in the explosion field.

Distributed testing has a long history. At its early stage in the $1970 \mathrm{~s}$, researchers began to research relevant distributed testing theories and successfully applied it to industrial automation test and control. In the 1980s, with the development of data communication technology and automatic test technology, the distributed testing system based on a bus structure was born [2] in which different instruments and testing modules could transmit information through the bus and be centrally controlled by the computer. Remote sensing technology can obtain surface information quickly and efficiently, so it plays an important part in the investigation of geological disasters [3]. Testing technology based on storage technology cannot meet the new requirements of distributed testing of a recent explosion area. To better complete the data transmission and node control of the distributed testing of a recent explosion area, the researchers at home started to integrate wireless communication technology into the distributed testing design [4].

Wireless LAN technology, as the product of a combination of a computer network and wireless communication, has the characteristics of easy installation, high-speed transmission, and scalability. The distributed testing system based on Wireless LAN technology became the focus of the recent study. The research, which relates to the distributed testing system, was conducted both in China and overseas. Literature [6] is a real-time study on the distributed system, which is based on a wireless local area network that provides the theoretical bases of the recently distributed system design of the wireless LAN.

Recently, the system, which is based on wireless distributed testing, has been widely studied overseas and has been applied to the testing of field signals, environmental monitoring, and other circumstances. There are two kinds of communication technology most widely used: one is wireless technology using the existing matured protocols, such as the technology of the Zigbee [5]; the other uses custom wireless protocols to transmit data. The technology of the Zigbee is hop internet technology, which is based on the wireless domain internet protocol and recently has been applied to the testing equipment mainly used for wireless trigger control rather than data transmission. Hop internet technology needs multiple nodes to transmit data indirectly; its slow transmission rate and the limited bandwidth are not fit for burst mode data transmission after an explosion. Relatively speaking, the custom wireless protocol cannot do the network architecture. It always uses signal point transmission rather than multi-point transmission, aiming at the distributed testing of the many testing nodes. A 
large number of testing nodes require data transmission, and this kind of the wireless transmission is not fit for distributed testing.

This paper presents a wireless distributed testing system based on WLAN. The system combined wireless communication technology with a distributed test system, making full use of the centralized management of the distributed test system and integrating the flexible character of being wireless. The system adopts Wireless LAN technology to connect all the testing nodes to the wireless local area network and realizes the monitoring, control and management in real-time, which makes it suitable for testing the complex field signal and competent for complex signal tests.

Because of the large loss of wireless nodes on testing nodes when near the ground, a general standard antenna, which is $2.4 \mathrm{G}$, will wear out when near the ground; therefore, this project designed a kind of high-gain antenna that will reduce the influence the ground has on it.

There is large amount of test data to gather in an explosion, so to improve transmission efficiency, a WLAN-based distributed measurement system using a star network topologic structure that consists of a data management center, field test nodes, and communication network was established.

\section{THE BASIC PRINCIPLES AND THE DESIGN OF THE DISTRIBUTED TESTING SYSTEM}

The distributed testing system is produced by the integration of the testing technology, communication technology and computer network technology, which uses the network as the communication means to test, measure and control the whole process and to apply to the testing technology of the area scattering. The distributed testing system refers to the computer measuring network system in which all the testing dots scatter in certain areas to connect the measuring equipment of a certain function, measuring the computer and controller through a LAN (Local Area Network, LAN) or the internet to realize the scatter operation, centralized management, collaborative work, information sharing and measuring process monitoring [7].

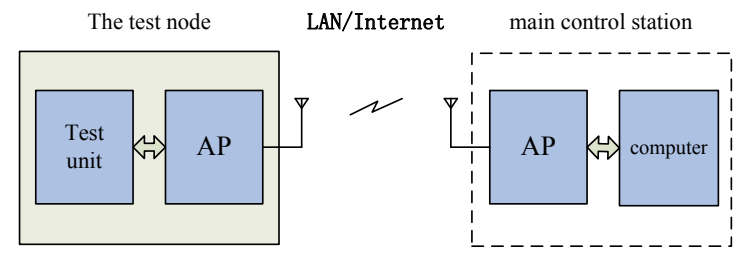

Figure 1. the forming framework of the distributed testing system

The wireless distributed testing system consists of three parts, which are the testing node, main control station, and the wireless local area network, which can communicate with each other. The structure is shown in Figure 1; the testing node should include the basic testing unit and network communication unit with functions such as signal collection and storage and wireless transmission. It is a professional integration intelligent instrument. The main control station should have the controlled monitoring testing nodes according to the software to exchange, show and deal with information and so on, and therefore, the software part should have the operation system, network protocol and so on. The wireless local area network, which consists of the wireless access point, wireless bridge, wireless antenna and other things, is the communication carrier of the testing node and the main control station.

In the distributed testing, a certain amount of testing nodes are displayed in the testing area, and all of them connect the main control station through the wireless local area network. In the test, the main control station can monitor and manage all states of the testing nodes through the wireless local area network and can collaborate to complete the test. In the process of testing, the main control station can read the testing data through the wireless local area network in real time, and when all the testing nodes receive the order, they will return the testing data to the main control station [8].

\section{THE DESIGN OF THE TESTING NODE}

\section{A. The forming and the working principles of the testing} node

The testing node of the testing transient pressure consists of the sensor, signal conditioning circuit, AD converter, FPGA, NAND flash, Wi-Fi wireless module and USB. The photo of the testing system is shown as the Figure 2.

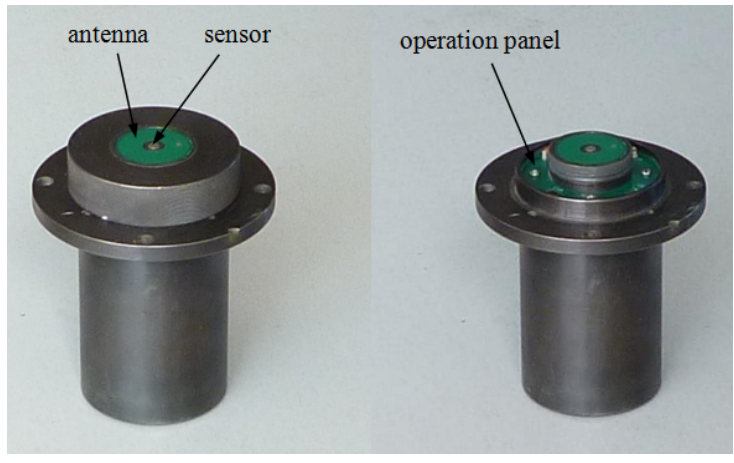

Figure 2. The photo of the testing system

The whole part selects the FPGA as the main control chip to control the logical timing. The NAND flash is suitable for storage of a large quantity data. The USB is used as a spare for data transmission to improve the reliability when the wireless communication breaks down or the wireless transmission is not needed. The flow chart of the testing nodes is shown in Figure 3. Its working process is that after the testing nodes power on, they enter the selecting state using a physical switch or the wireless control; otherwise they will be in a state of data waiting. After entering the signal selecting state, the system will continually store the data selected by the $\mathrm{AD}$ into the FIFO, and when the FIFO's one page is full, it will be stored into the NAND flash. When selecting the system signals, the FPGA monitors the data selected by the AD all the time, and once there is a meeting of the judging standard, it records the address of the NAND flash page. The judging standard mainly consists of two parts. The first one is the testing nodes set the threshold according to the explosive power and the signals greater than the set threshold in the monitoring; the second one is if the data of the $\mathrm{AD}$, which is monitored, expand rapidly. 


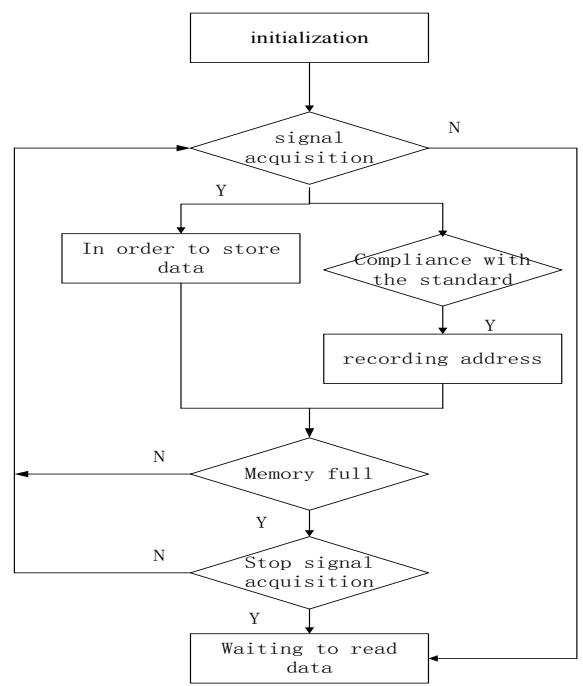

Figure 3. the flow chart of the testing system

The hardware structure is shown as Figure 4. The main control chip of the testing node selects the FPGA device of the Spartan6 series, which is low power consumption and low cost from the XILINX Company. It has the advantages of low power consumption, high performance, high speed, and a more comprehensive and rich interface, which meets the requirements of the application. It contains 9152 units; the card, which is $18 \mathrm{~Kb}$, has a Block RAM, a 100 customs IO pin and rich global clock recourse. The FPGA is used as the main control chip to select the storage signals and communicate with the remote control center at the same time meets the design requirements of the intelligent testing nodes.

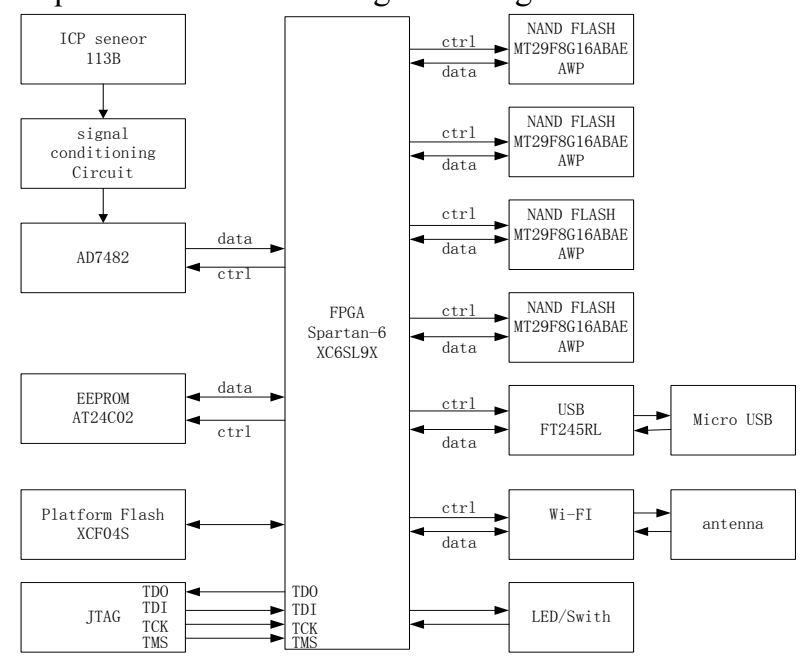

Figure 4. the hardware structure of the testing nodes

\section{$B$. The design of the wireless communication module}

In the design of these testing nodes, the WLAN module of the WizFi series from the WIZnet Company was selected. This series of modules supports the $802.11 \mathrm{~b} / \mathrm{g} / \mathrm{n}$, highly integrates 802.11 wireless communication contracts internally, and provides various serial interfaces. The baud rate of its interface is $921600 \mathrm{bps}$, which has the dynamic power management to realize low power consumption. The schematic diagram is shown as Figure 5. It supports the two different transmissions, TCP and UDP, and can be used under DHCP and fixed IP. This module has two different work modes and configuration modes that when the configuration parameters complete and enter the work mode, the user just has to interface communication to the data transmission. This module adopts the UART hardware interface to realize reliable transmission of the data under the control of the high speed transmission and the hardware flow control. It supports power on again, and then constructs the wireless network automatically according to the configuration parameters. When the wireless link disconnects, it will automatically link again. It supports the client and the server mode and can also realize the star network structure in which the AP is the center and peer-to-peer network structure. The use standard is the $802.11 \mathrm{~b} / \mathrm{g} / \mathrm{n}$ agreement, and its signal strength can automatically adjust the wireless rate [9].

\section{THE DESIGN OF WLAN}

The WLAN wireless module is the part by which the testing nodes access the wireless local area network, and its work is to communicate wirelessly according to the user's configuration parameters. Different wireless local area networks have different requirements. The configuration is also different. The configuration parameters of this design after overall consideration are as follows.

\section{(1)TCP setting.}

The TCP agreement is for the transport layer agreement of the connection, and in order to transmit the data reliably, it provides a series of data checks and retransmission mechanisms, it can realize effective control and multiplexing, and it can be used for data transmission, which requires high reliability.

\section{(2) The server and client setting}

The testing nodes are set as the server, and the remote control center as the client. In this way, the client can effectively control the testing nodes and the amount of the data set, avoiding any jam caused by the network. Meanwhile, the testing nodes are used as the server, monitoring the requests of the client, responding to the connection requests of the client, and establishing connections.

(3) IP address and the port number.

This project selected a static IP setting in that every testing node only has one IP address and port number. When the remote control center communicates with the testing nodes, the client software can communicate through socket technology by the IP address and the port number 


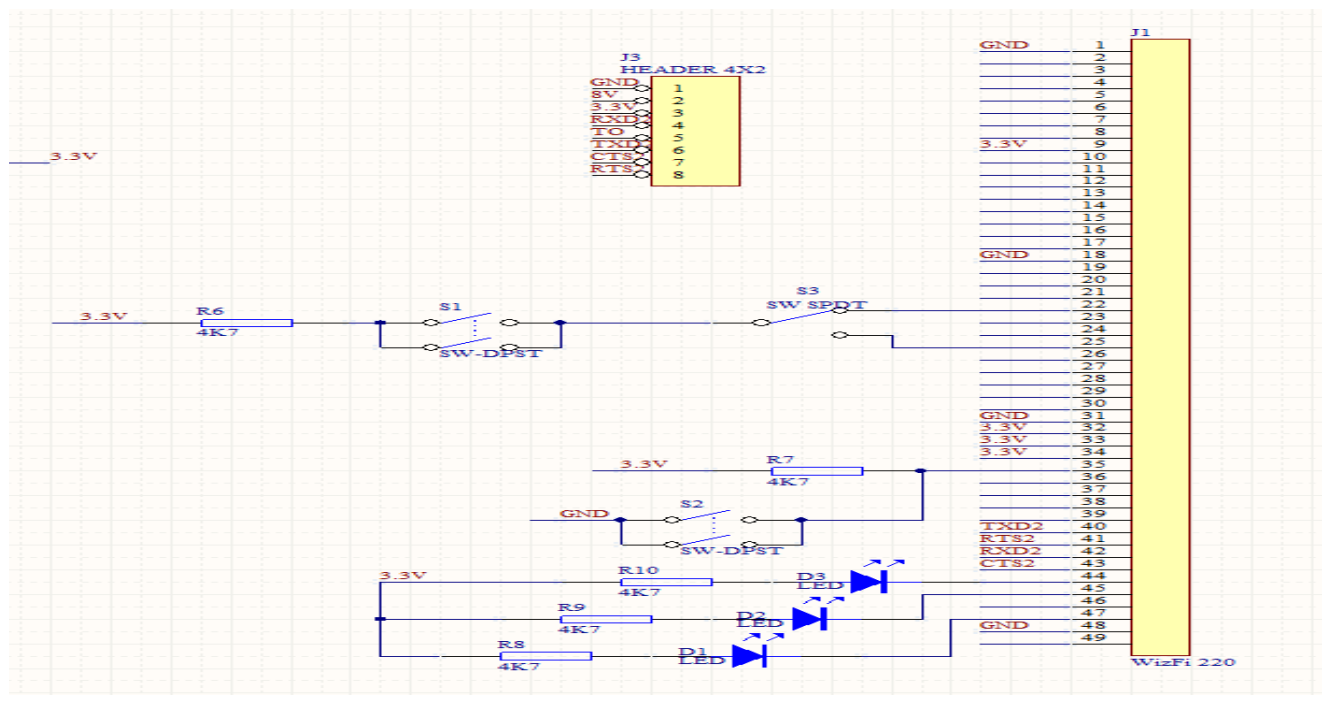

Figure 5. the schematic diagram of the wireless communication module

\section{THE DESIGN OF THE WIRELESS LOCAL AREA NETWORK}

According to the design process of the wireless local area network, it finally forms the wireless local area network design scheme of the explosion area distributed testing. Its make-up is shown as Figure 6. The front AP completes the cover of the wireless local area network in which all the testing nodes are linked to the front AP to connect the wireless local area network. The front AP connects the repeater bridge through the cable to realize long distance transmission. The bridge selects the point to point transmission mode, and when using the antenna, the repeater bridge should make sure of the direction of the antenna alignment, otherwise it will affect the distance of the transmission. The transmission distance is set at $2 \mathrm{~km}$ in this system. The remote bridge selects the method of using the cable to connect the client and the network interface of the client's PC. In the whole system, the AP and bridge all select the power supply mode of the POE and the lithium battery as the power supply. The front AP selects the high power RocketM2 and the AM-2G15-120 of the high-gain antenna and selects the fixed channel set, avoiding the same frequency interface as the wireless bridge. The wireless bridge selects NSM2 with a small size integrated antenna, and the longest distance of the transmission is $15 \mathrm{~km}$. It is suitable for wireless data transmission of a long distance. All of the IP addresses of the wireless local area network select a static setting. Its IP address is in the same segment with the testing nodes, and in this way, the remote control center can manage all the testing nodes through the client. Every testing node has only one IP address to manage and control it conveniently [11].

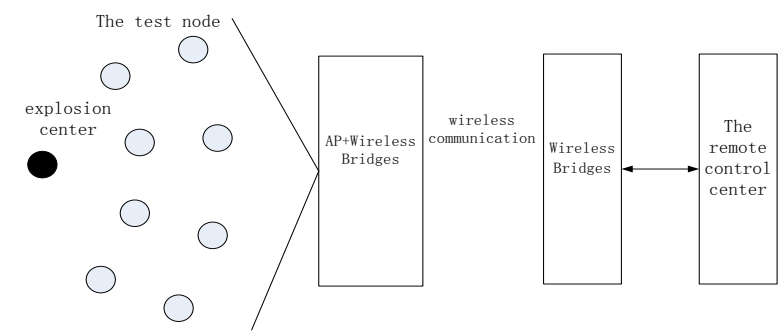

Figure 6. the network scheme of the wireless local area network

\section{THE REMOTE CONTROL CENTER}

The remote control center mainly consists of the PC machine and the client software. The PC machine is the hardware platform of the client software, and in using its powerful hardware resource, it has better processing ability and its operation system is an integrated network protocol, which can has easy access to the internet and local area network through the network interface or a wireless network card $[12,13]$. The design of the client software selects the LabVIEW platform to develop, mainly aiming at the centralized control and management of each testing node, collecting the data of the testing node, invoking the relevant program to process the testing data and so on. The remote control center connects with the AP through the network interface, inserts into the whole wireless local area network of the distributed testing system, and then controls the whole distributed testing system. The client selects the TCP/IP agreement, and the technology development in the LabVIEW pl atom. In the distributed testing system based on the $\mathrm{C} / \mathrm{S}$ framework, the remote control center is the client. The client software integrates all the controlling orders; the AP transmits the order to control the testing nodes. All of the functions of the client software are shown in Figure 7: (1) the monitoring state of all the testing nodes, (2) the setting of the parameters of the testing nodes, (3) the wireless control, and (4) the recycle and processing of the data. 
PAPER

A Distributed Remote Real-time Pressure Measuring System Based on Wireless Communication Tech...

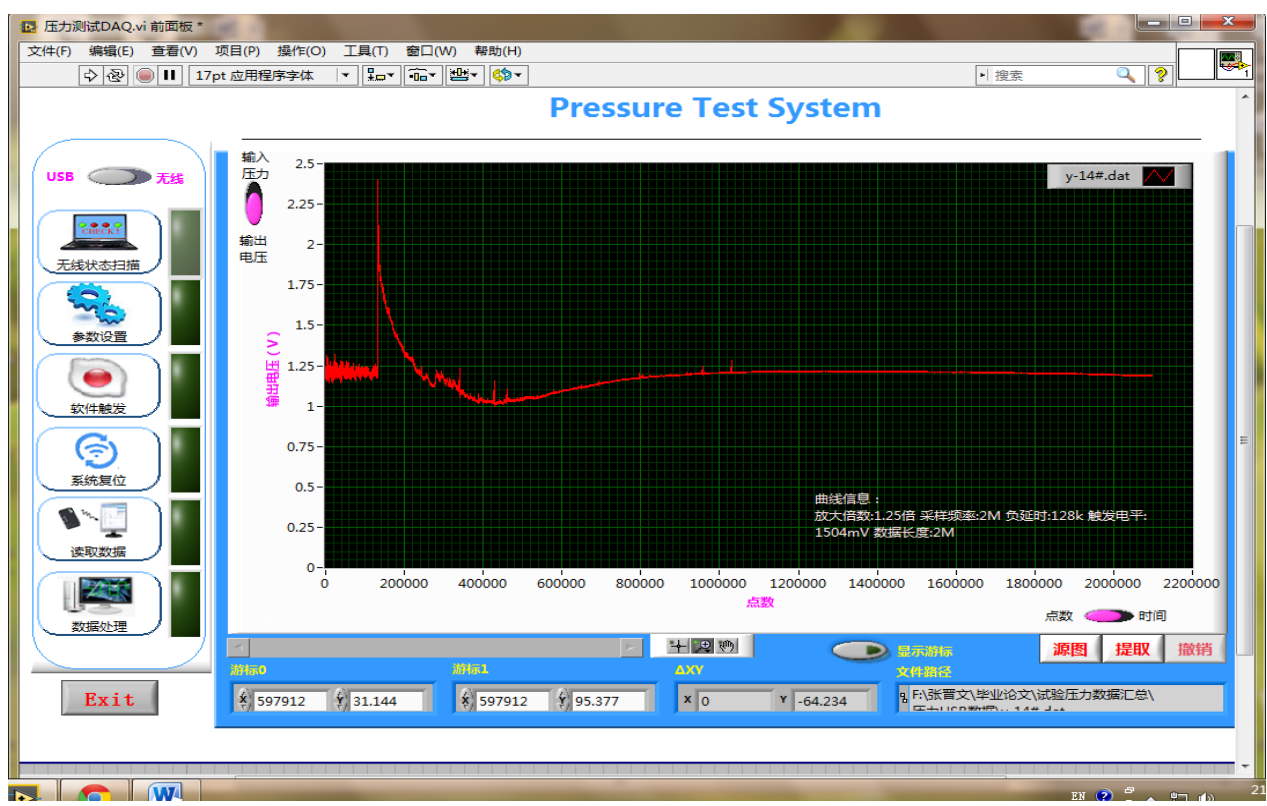

Figure 7. the client software

\section{THE PERFORMANCE TESTING}

In order to test the performance of the testing system, 16 sets of the equipment were networked; all the testing nodes were laid out in a radius of $10 \mathrm{~m}, 20 \mathrm{~m}$ and $30 \mathrm{~m}$ and buried deep with the antenna and surface at the same level, the covering AP connects the bridge by the cable, and it stands 6 meters high. Its fan antenna leaned at an angle of about $2^{\circ} \mathrm{C} \sim 3^{\circ} \mathrm{C}$; the remote client $\mathrm{PC}$ connected the bridge; the bridge was 6meters high, which is about $2 \mathrm{~km}$ away from the AP, as shown in Figure 8. The distance of the AP and the testing nodes determined the cover area and location of the AP, and this experiment mainly verified the limits of the communication distance of the testing nodes and the AP.

After the testing nodes were linked to the covering AP and the wireless bridge was networked successfully, the remote client PC then set the parameters, back read the parameters, selected synchronous and stop selecting at the same time, and after that, made a burst-mode data transmission of $1 \mathrm{MB}$. The transmission performance of the wireless bridge was verified by all the testing nodes' real-time communication. Diagram 1 shows the results after several times of testing.

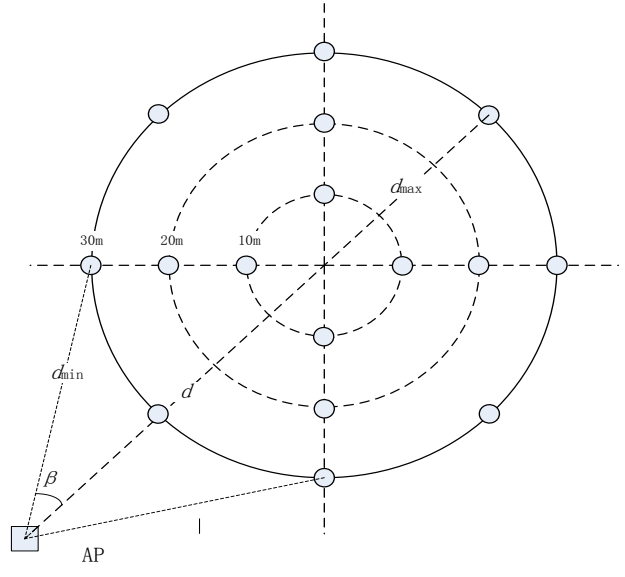

Figure 8. the node layout diagram
TABLE I.

THE TESTING RESULTS OF THE COVERED DISTANCE

\begin{tabular}{|c|c|c|c|}
\hline $\begin{array}{c}\text { linear } \\
\text { distance( } \\
\text { m) }\end{array}$ & $\begin{array}{c}\text { The range of } \\
\text { the signal } \\
\text { strength(dBm) }\end{array}$ & $\begin{array}{c}\text { The } \\
\text { transmissi } \\
\text { on time(s) }\end{array}$ & $\begin{array}{c}\text { Transmission } \\
\text { rate(kbps) }\end{array}$ \\
\hline $100 \mathrm{~m}$ & $-54 \sim-58$ & 48 & 165 \\
\hline $150 \mathrm{~m}$ & $-59 \sim-64$ & 48 & 165 \\
\hline $200 \mathrm{~m}$ & $-72 \sim-76$ & 50 & 168 \\
\hline $250 \mathrm{~m}$ & $-84 \sim-87$ & 54 & 150 \\
\hline
\end{tabular}

(1)When the AP is $150 \mathrm{~m}$ away from the testing center, $d=150 \mathrm{~m}, d_{\max }=180 \mathrm{~m}, d_{\min }=160 \mathrm{~m}$ at present $\beta=7.6^{\circ}$. All of the testing nodes in the test could establish wireless access with the AP, which could also be realized under the wireless control order. In the burst-mode transmission of the data, all of the data of the testing nodes could be back read at full speed.

(2)When the AP is $200 \mathrm{~m}$ away from the testing center, $d=200 \mathrm{~m}, d_{\max }=230 \mathrm{~m}, d_{\min }=180 \mathrm{~m}, \beta=6.8^{\circ}$. The testing nodes in the test could establish wireless connection with the AP, which could also be realized under the wireless order. But in the burst-mode transmission of the data, the testing nodes could not be back read at the same time and the reading process caused obvious data loss. When decreased to 5-7, it could better complete the data transmission.

\section{CONCLUSIONS}

The wireless distributed testing system is the combination of the storage testing technology and the wireless transmission technology and centrally controls, manages and monitors all the testing nodes with independent function, completely solving the problem of complicated operations caused by highly scattered testing nodes and a large amount of information. And it has already been the main technical scheme of data transmission and control. The technical scheme has remarkable superiority for completing complicated and remote tasks, it makes high-speed transmission and handle data efficiently possible.The wireless distributed testing system is advantageous, especially in the high temperature, 
PAPER

A Distributed Remote Real-time Pressure MeAsuring System Based on Wireless Communication Tech...

high pressure, harsh electromagnetic, and other complicated environments, and has broad prospects for application.

\section{REFERENCES}

[1] Guirao C. M. and Bach G. G, "Non-self-similar blast waves," Combustion, Explosion and Shock Waves, vol. 27,no.3, pp.342351,1991. http://dx.doi.org/10.1007/BF00789669

[2] S. Wan and Y.Z. Fan, "Design of a remote control vehicle based wireless communication used for fighting fire," International Journal of Online Engineering, vol. 9, no.5, pp. 79-82, 2013. http://dx.doi.org/10.3991/ijoe.v9iS6.2891

[3] Grimaidi. Domenico, "Distributed measurement system," Measurement Journal of The International, vol. 30,no.7, pp.279287,2001 .

[4] Luo X, Liu D, and Ye D, "Multi-agent based distributed measurement systems in CORBA environment," IEEE Instrumentation and Measurement Technology Conference, vol.2,no.8, pp.821-826,2001.

[5] Xianfeng ZHENG and Zheng FAN, "Design of the zigbee technology-based wireless sensor network for earth temperature monitoring," International Journal of Online Engineering, vol. 10, no.5, pp. 63-67, 2014. http://dx.doi.org/10.3991/ ijoe.v10i5.4034

[6] Li jinwen and Ma Boyuan, "Remote Monitoring in Distributed Measurement System Using DataSocket JavaBean and Java Applet,"Advanced Materials Research, vol. 476,no.3, pp.20323035,2012

[7] De La Rosa and Juan José González, "A web-based distributed measurement system for electrical power quality assessment,"Journal of the International Measurement Confederation,vol. 6,no.3, pp.771-780,2010.

[8] Ciancetta Fabrizio, Fiorucci Edoardo, and Gallo Daniele, "A Web Service interface for a distributed measurement system based on decentralized sharing network," Sensors and Transducers,vol.6,no.10, pp.209-218,2013.
[9] L Skrzypczak, D Grimaldi, and R Rak, "Analysis of the different wireless transmission technologies in distributed measurement systems,"IEEE International Workshop on Intelligent Data Acquisition and Advanced Computing Systems,vol.2,no.7, pp.673678,2009. http://dx.doi.org/10.1109/idaacs.2009.5342890

[10] A. Aiello, D.L. Cami, D. Grimaldi, and G. Guglielmelli, "Wireless distributed measurement system by using mobile devices,"IEEE Intelligent Data Acquisition and Advanced Computing Systems,vol.9,no.8, pp.316-319, 2005. http://dx.doi.org/10.1109/ IDAACS.2005.282994

[11] Ciancetta, Fabrizio, Bucci, and Giovanni, D'Apice Biagio, "A distributed measurement system based on Peer-To-Peer architecture for River Monitoring,"Virtual Environments HumanComputer Interfaces and Measurement Systems,vol.4,no.4, pp.8792,2007 .

[12] Rappaport T. S, "Wireless communications: principles and practice," New York: Prentice Hall, vol.2,no.5, pp.152-156, 2001.

[13] Salman M.K., Ahmad R.B., Yahya Abid, "A new approach for efficient utilization of resources in WiMAX cellular networks," Tehnicki Vjesnik, vol.21, no.6, pp. 1385-1393, 2014.

\section{AUTHORS}

Wang Wei is with the North University of China. He is now with the School of Instrument and Electronics and working toward a doctor's degree. His scientific research interests are concerned with digital signal processing in dynamic measurement and uncertainty of measurement systems.

Zhang Zhijie is with the North University of China. He is a full professor with the School of Instrument and Electronics. He has teaching experience of 30 years and has completed ten scientific research projects.

Submitted 24 April 2015. Published as resubmitted by the authors 20 August 2015. 